\title{
APPROXIMATION OF SET-VALUED FUNCTIONS WITH COMPACT IMAGES-AN OVERVIEW
}

\author{
NIRA DYN and ELZA FARKHI \\ School of Mathematical Sciences, Sackler Faculty of Exact Sciences \\ Tel-Aviv University, Ramat-Aviv, 69978 Tel Aviv, Israel \\ E-mail:niradyn@post.tau.ac.il,elza@post.tau.ac.il
}

\begin{abstract}
Continuous set-valued functions with convex images can be approximated by known positive operators of approximation, such as the Bernstein polynomial operators and the Schoenberg spline operators, with the usual sum between numbers replaced by the Minkowski sum of sets. Yet these operators fail to approximate set-valued functions with general sets as images. The Bernstein operators with growing degree, and the Schoenberg operators, when represented as spline subdivision schemes, converge to set-valued functions with convex images. To obtain approximating operators for set-valued functions with general images, we use a binary average between sets, termed the "metric average", which was introduced by Artstein for the construction of piecewise linear interpolants to set-valued functions. Representing each of the above mentioned operators in terms of repeated binary averages, and replacing the binary average between numbers by the metric average, we obtain operators for set-valued functions with compact images. In case of the Schoenberg operators, represented either by the de Boor algorithm or by spline subdivision schemes, the operators are approximating. In case of the Bernstein operators, the convergence with the increase of the degree is established only for set-valued functions with $1 \mathrm{D}$ images, consisting of the same number of intervals.
\end{abstract}

1. Introduction. The paper surveys our work on the approximation of set-valued functions (SVFs) with compact images in $\mathbb{R}^{n}$ by set-valued operators extending some positive linear operators for real-valued functions $[9,10,11,13]$. The operators considered are the Schoenberg spline operators in explicit form or as the limit of subdivision schemes, and extensions of the Bernstein polynomial operators.

The adaption of these operators to set-valued functions requires replacing operations between numbers by operations between sets. The most natural definition of Minkowski sum of sets is a proper substitute for addition of numbers, only in case of SVFs with convex images. In this case, the well-known representation of convex compact sets in

2000 Mathematics Subject Classification: 26E25, 41A36, 41A65, $47 \mathrm{H} 04$.

The paper is in final form and no version of it will be published elsewhere. 
terms of their support functions defines an embedding of the cone of convex compact subsets of $\mathbb{R}^{n}$ into a Banach space with an addition operation induced by the Minkowski sum of sets and a norm generated by the Hausdorff distance between sets. Thus the approximation of SVFs reduces to the approximation of their support functions. The application of known approximation results from the case of real-valued functions to the case of SVFs with convex compact images is possible $[18,7,2,9]$. The positivity of the operators ensures that the images of the approximating SVFs do not leave the cone of convex compact sets, and some of the operators preserve convexity of the graph and set-inclusion [9].

If the images of the approximated SVF are not convex, it was noticed by Vitale [18] that the usual approximating positive operators with Minkowski sums of sets fail to approximate, and a phenomenon of convexification appears in case of the Bernstein operators [18], and for some Bernstein-type operators [11]. Also positive subdivision schemes map initial compact sets to a limit SVF with convex images identical to the limit SVF obtained from the convex hulls of the initial sets [11]. This phenomenon is similar to the well-known convexity of the Aumann integral, when regarded as a limit of MinkowskiRiemann sums of sets, even if the integrated SVF does not have convex images (see e.g. [11]). The obvious conclusion is that operators with Minkowski sums of sets are not effective for the approximation of SVFs with general compact images.

An alternative operation for the average of two sets is defined by Artstein [1]. This operation has an important metric property with respect to the Hausdorff metric, which ensures that the piecewise-linear set-valued interpolant is approximating continuous SVFs [1]. The lack of associativity in this operation is the reason why it is hard to extend this binary operation to an average of three or more sets. To use this operation in the adaptation of positive operators to SVFs, we need to represent these operators in terms of repeated binary averages. Note that every linear operator, which is exact for constants and is a linear combination function's values, can be represented in terms of repeated binary averages in more than one way [19].

For the evaluation of the Schoenberg operators we use either the de Boor algorithm ([4], Chapter X), or spline subdivision schemes represented in terms of repeated binary averages [10]. The Bernstein operator may be represented in terms of repeated binary averages by the De Casteljeau algorithm [14]. The Schoenberg operators so defined approximate set-valued functions which are Hölder continuous [10, 13], while for the Bernstein operators approximation is proved only for Hölder continuous set-valued functions with images in $\mathbb{R}$ all of the same topology [13]. It is not clear whether the so defined Bernstein operators approximate SVFs with images in $\mathbb{R}^{n}$ for $n>1$.

The paper is organized as follows. After presenting definitions and notations in the next section, we recall some basic facts on positive linear operators for real-valued functions in Section 3. Section 4 discusses approximations of SVFs with convex images by Schoenberg operators, spline subdivision schemes and Bernstein-type operators. Section 5 is devoted to the approximation of SVF with compact (not necessarily convex) images. After showing that the above operators with Minkowski sums do not approximate, we study them when defined in terms of metric averages of sets. 
2. Preliminaries. First we introduce some notation. The collection of all univariate polynomials of degree less than or equal to $m$ is denoted by $\pi_{m}$, the collection of all nonempty compact subsets of $\mathbb{R}^{n}$ is denoted by $\mathcal{K}_{n} \cdot \mathcal{C}_{n}$ denotes the collection of convex sets in $\mathcal{K}_{n},\langle\cdot, \cdot\rangle$ is the inner product in $\mathbb{R}^{n},|x|$ is the Euclidean norm of $x \in \mathbb{R}^{n}, S_{n-1}$ is the unit sphere in $\mathbb{R}^{n}$, co $A$ denotes the convex hull of the set $A$.

The Hausdorff distance between two sets $A$ and $B$ in $\mathbb{R}^{n}$ is defined by

$$
\operatorname{haus}(A, B)=\max \left\{\sup _{x \in A} \operatorname{dist}(x, B), \sup _{y \in B} \operatorname{dist}(y, A)\right\},
$$

where $\operatorname{dist}(x, A)=\inf \{|x-y|: y \in A\}$. By $|A|=\sup \{|x|: x \in A\}$ we denote the "norm" of the set $A$. Let us recall that $\mathcal{K}_{n}$ is a complete metric space with respect to the Hausdorff metric [16].

The support function $\delta^{*}(A, \cdot): \mathbb{R}^{n} \rightarrow \mathbb{R}$ is defined for $A \in \mathcal{K}_{n}$ as

$$
\delta^{*}(A, l)=\max _{a \in A}\langle l, a\rangle, \quad l \in \mathbb{R}^{n} .
$$

The set of all projections of $x$ on the set $A \in \mathcal{K}_{n}$ is $\Pi_{A}(x)=\{a \in A:|a-x|=\operatorname{dist}(x, A)\}$. The set difference of $A, B \in \mathcal{K}_{n}$ is $A \backslash B=\{a: a \in A, a \notin B\}$. A linear Minkowski combination of the sets $A_{1}, A_{2}, \ldots, A_{k}$ is

$$
\sum_{i=1}^{k} \lambda_{i} A_{i}=\left\{\sum_{i=1}^{k} \lambda_{i} a_{i}: a_{i} \in A_{i}, i=1, \ldots, k\right\},
$$

for $A_{i} \in \mathcal{K}_{n}$ and $\lambda_{i} \in \mathbb{R}$. The Minkowski sum $\sum_{i=1}^{k} A_{i}$ corresponds to a linear Minkowski combination with $\lambda_{i}=1$. A Minkowski average (a Minkowski convex combination) of sets is a linear Minkowski combination with $\lambda_{i}$ non-negative, summing up to 1 .

It is easy to verify that for a general set $A$,

$$
\lambda A+(1-\lambda) A \supset A, \quad \lambda \in \mathbb{R}
$$

while for a convex set,

$$
\lambda A+(1-\lambda) A=A, \quad 0 \leq \lambda \leq 1
$$

The notions convergence, continuity, Hölder continuity for set-valued functions are to be understood with respect to the Hausdorff metric (distance), e.g. the SVF, $F(\cdot)$, is Hölder continuous with exponent $\alpha$ if

$$
\operatorname{haus}\left(F\left(t_{1}\right), F\left(t_{2}\right)\right) \leq C\left|t_{1}-t_{2}\right|^{\alpha}
$$

with a constant $C$ which depends on $F$. The collection of Hölder continuous SVFs with exponent $\alpha$ is denoted by $\mathcal{H}_{\alpha}$. Clearly, $\mathcal{H}_{\alpha}$ contains the Hölder continuous real-valued functions with exponent $\alpha$.

For a real-valued function $f: I \rightarrow \mathbb{R}, I \subset \mathbb{R}$, we denote by $\operatorname{supp}(f)$ the support of $f$. The notions of moduli of continuity and smoothness of such $f$ are central to our paper (see e.g. [6], Chapter 2). The modulus of continuity of $f$ with a step $\delta \geq 0$, is $\omega_{I}(f, \delta)=\sup _{0<h \leq \delta}\left\|\Delta_{h, I}(f, \cdot)\right\|_{\infty}$, with

$$
\Delta_{h, I}(f, t)= \begin{cases}f(t+h)-f(t) & \text { for } t, t+h \in I, \\ 0 & \text { otherwise. }\end{cases}
$$

$\omega_{I}(f, \delta)$ is also known as the first modulus of smoothness. 
The second modulus of smoothness is $\omega_{2, I}(f, \delta)=\sup \left\{\left\|\Delta_{h, I}^{2}(f, \cdot)\right\|_{\infty}: 0<h \leq \delta\right\}$, with $\Delta_{h, I}^{2}(f, t)=\Delta_{h, I}\left(\Delta_{h, I}(f, t), t\right)$.

We use in this paper the following properties of these moduli:

1. For $f \in \mathcal{H}_{\alpha}, \omega_{I}(f, \delta)=O\left(\delta^{\alpha}\right)$.

2. $\omega_{I}(f, \lambda \delta) \leq(\lambda+1) \omega_{I}(f, \delta)$.

3. If $f$ is continuously differentiable on $I$, then $\omega_{2, I}(f, \delta) \leq \delta \omega_{I}\left(f^{\prime}, \delta\right)$.

3. Some positive linear operators for real-valued functions. In this section we review results on the approximation of real-valued functions by special positive linear operators. These results are applied later to the approximation of set-valued functions with convex compact images. We consider linear operators of the form

$$
\mathcal{A}_{N}(f, t)=\sum_{i=0}^{N} C_{i, N}(t) f\left(t_{i}^{N}\right),
$$

applied to real-valued functions on $[0,1]$, where $t_{0}^{N}<t_{1}^{N}<\ldots<t_{N}^{N}$ are given points in $[0,1]$ and $C_{i, N}(t) \geq 0$. Furthermore, we require that $\sum_{i=0}^{N} C_{i, N}(t)=1$ either in $[0,1]$, or in most of it. We restrict this class to operators which approximate continuous functions in $[0,1]$ or in most of it.

We recall that a linear operator $L(f, x)$ is a positive operator if for a non-negative $f, L(f, x)$ is non-negative. Clearly, $\mathcal{A}_{N}$ is a positive linear operator. It reproduces the constant functions, namely $\mathcal{A}_{N}(f, t)=f(t)$ for $f(t)=$ const for all $t$ if $\sum_{i=0}^{N} C_{i, N}(t)=1$. In fact, $\mathcal{A}_{N}(f, t)$ is a weighted average of the function values $f\left(t_{i}^{N}\right), i=0,1, \ldots, N$.

Here are some examples of such positive linear operators.

3.1. Schoenberg operators. Let $t_{i}^{N}=\frac{i}{N}, i=0,1, \ldots, N$. Consider the operators (2) with the coefficients $C_{i, N}^{m}(t)=B_{m}(N t-i)$ or $C_{i, N}^{m}(t)=\tilde{B}_{m}(N t-i)$, where $B_{m}(\cdot)$ is a B-spline of degree $m$ (order $m+1$ ) with integer knots and support [0,m+1], and where $\tilde{B}_{m}(t)=B_{m}\left(t-\frac{m+1}{2}\right)$. We denote the above two spline operators of degree $m$ by

$$
S_{m}^{N}(f, t)=\sum_{i=0}^{N} B_{m}(N t-i) f\left(t_{i}^{N}\right), \quad \tilde{S}_{m}^{N}(f, t)=\sum_{i=0}^{N} \tilde{B}_{m}(N t-i) f\left(t_{i}^{N}\right),
$$

and call $S_{m}^{N}(f, t)$ the classical Schoenberg operator, and $\tilde{S}_{m}^{N}(f, t)$ the symmetric Schoenberg operator. An important special case is the piecewise-linear interpolant $\tilde{S}_{1}^{N}$ with

$$
\tilde{B}_{1}(t)= \begin{cases}1-|t| & \text { for }|t| \leq 1, \\ 0 & \text { otherwise. }\end{cases}
$$

Note that the symmetric Schoenberg operators reproduce constant and linear functions, namely $\tilde{S}_{m}^{N}(f, \cdot)=f(\cdot)$ for any $f \in \pi_{1}$, only on the sub-interval $\left[\tilde{a}_{N}, \tilde{b}_{N}\right]=\left[\frac{m-1}{2 N}, 1-\right.$ $\left.\frac{m-1}{2 N}\right]$. It is easy to verify that the classical Schoenberg operators reproduce constants on $\left[a_{N}, b_{N}\right]=\left[\frac{m}{N}, 1\right]$, but not linear functions.

We recall two results on the approximation by linear operators of local character, and apply them later to the Schoenberg spline approximation (cp. [6], Chapter 7, Lemma 7.1 and Theorem 7.2). For the sake of completeness we provide short proofs. 
Result A. Let $f:[0,1] \rightarrow \mathbb{R}$ be continuous and let $L_{N}(f, t)=\sum_{i=0}^{N} f\left(\frac{i}{N}\right) \psi(N t-i)$, with $\psi$ a continuous function of compact support. If $\psi$ is positive on the interior of $\operatorname{supp}(\psi)$ and $\sum_{i=0}^{N} \psi(N t-i)=1$ for $t \in\left[a_{N}, b_{N}\right] \subset[0,1]$, then

$$
\left\|f-L_{N}(f, \cdot)\right\|_{\infty,\left[a_{N}, b_{N}\right]} \leq C \omega_{[0,1]}\left(f, \frac{1}{N}\right),
$$

where $C$ depends on $|\operatorname{supp}(\psi)|$.

Proof. For a fixed $t \in\left[a_{N}, b_{N}\right], \psi(N t-i)>0$ for $i \in I_{t}=\left\{i \in \mathbb{Z}: t-\frac{i}{N} \in \frac{1}{N} \operatorname{supp}(\psi)\right\}$, and we get

$$
\begin{aligned}
L_{N}(f, t)-f(t) & =\sum_{i \in I_{t}}\left(f\left(\frac{i}{N}\right)-f(t)\right) \psi(N t-i) \\
& \leq \omega_{[0,1]}\left(f, \frac{|\operatorname{supp}(\psi)|}{N}\right) \leq C \omega_{[0,1]}\left(f, \frac{1}{N}\right) .
\end{aligned}
$$

RESUlt B. Under the conditions of Result $A$, if $L_{N}$ reproduces also linear functions on $\left[a_{N}, b_{N}\right]$, then, for $f \in C^{2}[0,1]$,

$$
\left\|f-L_{N}(f, \cdot)\right\|_{\infty,\left[a_{N}, b_{N}\right]} \leq \frac{C}{N^{2}}\left\|f^{\prime \prime}\right\|_{\infty,[0,1]},
$$

where $C$ depends on $|\operatorname{supp}(\psi)|$.

Proof. For a fixed $t \in\left[a_{N}, b_{N}\right]$, let $g(x)=f(t)+(x-t) f^{\prime}(t)$ be the linear Taylor approximation of $f$ at $t$. Then $f(x)-g(x)=f^{\prime \prime}(\xi) \frac{(x-t)^{2}}{2}$ with $\xi$ in the interval with end points $x, t$. Since $L_{N}(g, \cdot)=g$ and $g(t)=f(t)$, we get by the linearity of $L_{N}$,

$$
L_{N}(f, t)-f(t)=L_{N}(f-g, t)=\sum_{i \in I_{t}}(f-g)\left(\frac{i}{N}\right) \psi(N t-i) .
$$

Since $|t-i / N| \leq|\operatorname{supp}(\psi)| / N$, we get $|(f-g)(i / N)| \leq \frac{1}{2}\left|f^{\prime \prime}\left(\xi_{i}\right)\right|(|\operatorname{supp}(\psi)| / N)^{2}$, with $\xi_{i} \in(0,1)$, and $(6)$ follows.

For the Schoenberg operators applied to Hölder continuous real-valued functions, it follows from Result A that

$$
\begin{aligned}
& \left|S_{m}^{N}(f, t)-f(t)\right|=O\left(\frac{1}{N^{\alpha}}\right), \quad t \in\left[\frac{m}{N}, 1\right], \\
& \left|\tilde{S}_{m}^{N}(f, t)-f(t)\right|=O\left(\frac{1}{N^{\alpha}}\right), \quad t \in\left[\frac{m-1}{2 N}, 1-\frac{m-1}{2 N}\right],
\end{aligned}
$$

where $\alpha$ is the Hölder exponent of $f$.

From Result B one gets for the symmetric Schoenberg operators applied to a realvalued function with a bounded second derivative that

$$
\left|\tilde{S}_{m}^{N}(f, t)-f(t)\right|=O\left(\frac{1}{N^{2}}\right) .
$$

A weaker approximation result holds for $f$ such that $f^{\prime} \in \mathcal{H}_{\alpha}$, namely

$$
\left|\tilde{S}_{m}^{N}(f, t)-f(t)\right|=O\left(\frac{1}{N^{1+\alpha}}\right) .
$$


3.2. Spline subdivision schemes. For real-valued functions the Schoenberg operators can be evaluated by subdivision schemes (see e.g. [8]).

Given the initial sequence $f_{i}^{0}=f\left(\frac{i}{N}\right), i=0, \ldots, N$ of values in $\mathbb{R}$, with $f_{i}^{0}=0$ for $i \in \mathbb{Z} \backslash\{0,1, \ldots, N\}$, the spline subdivision scheme for the evaluation of $S_{m}^{N}(f, \cdot)$ is given by the refinement steps

$$
f_{i}^{k}=\sum_{j \in \mathbb{Z}} a_{i-2 j}^{[m]} f_{j}^{k-1}, \quad i \in \mathbb{Z}, \quad k=1,2, \ldots
$$

with the mask $a_{i}^{[m]}=\left(\begin{array}{c}m+1 \\ i\end{array}\right) / 2^{m}, i=0,1, \ldots, m+1$ and $a_{i}^{[m]}=0$ for $i \in \mathbb{Z} \backslash\{0,1, \ldots, m+1\}$.

At the $k$-th refinement level one defines the piecewise linear function

$$
f^{[k]}(t)=\sum_{i \in \mathbb{Z}} f_{i}^{k} \tilde{B}_{1}\left(2^{k} t-i\right), \quad t \in \mathbb{R}
$$

where $\left\{f_{i}^{k}, i \in \mathbb{Z}\right\}$ are the values generated by the subdivision scheme at refinement level $k$. The scheme (11) is uniformly convergent, namely the sequence $\left\{f^{[k]}(\cdot)\right\}_{k \geq 0}$ is a Cauchy sequence, and its limit function is of the form (see e.g. [8])

$$
f^{\infty}(t)=S_{m}^{N}\left(f, N^{-1} t\right) .
$$

For $m$ odd and the shifted spline weights $\tilde{a}_{i}^{[m]}=a_{i-\frac{m+1}{2}}^{[m]}, i=0,1, \ldots, m+1$, the limit of the subdivision scheme is $\tilde{S}_{m}^{N}\left(f, N^{-1} \cdot\right)$.

3.3. Bernstein-type operators. Bernstein-type operators of a function $f:[0,1] \rightarrow \mathbb{R}$ are defined by

$$
\mathcal{B}_{N}^{\chi}(f, t)=\sum_{i=0}^{N} P\left(\chi_{t}^{N}=i\right) f\left(\frac{i}{N}\right),
$$

where for a given $N \in \mathbb{N}$ and $t \in[0,1], \chi_{t}^{N}$ is a discrete random variable with values $i \in\{0,1, \ldots, N\}$, and expectation

$$
E\left(\chi_{t}^{N}\right)=N t
$$

In the classical Bernstein polynomials of degree $N, \chi_{t}^{N}$ is a binomially distributed random variable with $C_{i, N}(t)$ in (2) given by $P\left(\chi_{t}^{N}=i\right)=\left(\begin{array}{c}N \\ i\end{array}\right) t^{i}(1-t)^{N-i}$. Thus

$$
B_{N}(f, t)=\sum_{i=0}^{N}\left(\begin{array}{c}
N \\
i
\end{array}\right) t^{i}(1-t)^{N-i} f\left(\frac{i}{N}\right) .
$$

Another example of a Bernstein-type operator is the polynomial approximation of degree $N$, defined by $\chi_{t}^{N}$ the random variable with the hypergeometric distribution with parameters $M \geq N$,

$$
P\left(\chi_{t}^{N}=i\right)= \begin{cases}\frac{\left(\begin{array}{c}
M t \\
i
\end{array}\right)\left(\begin{array}{c}
M(1-t) \\
N-i
\end{array}\right)}{\left(\begin{array}{c}
M \\
N
\end{array}\right)} & \text { for } N-M(1-t) \leq i \leq M t \\
0 & \text { otherwise. }\end{cases}
$$

Here the real binomial coefficients are $\left(\begin{array}{l}y \\ k\end{array}\right)=\frac{y(y-1) \ldots(y-k+1)}{k !}$.

Note that the requirement (15) implies that the Bernstein-type operators reproduce linear functions. The following approximation theorem is applicable in this case. 
Result C (see [6], Chapter 9, Theorems 4.3, 4.4). Let $L_{N}(f, \cdot), N=1,2, \ldots$ be a sequence of positive linear operators on the space of continuous functions defined on $[0,1]$, which reproduce linear functions. Denote $u_{2}(x)=x^{2}$, and

$$
\lambda_{N}(x)=\left|L_{N}\left(u_{2}, x\right)-u_{2}(x)\right| .
$$

Then, for any continuous function $f:[0,1] \rightarrow \mathbb{R}$,

$$
\left|f(x)-L_{N}(f, x)\right| \leq K \omega_{2,[0,1]}\left(f, \sqrt{\lambda_{N}(x)}\right),
$$

where $K$ does not depend on $N$.

Now, suppose that $\lambda_{N}(x) \leq \Lambda(x) / N^{\beta}$ for the operator in (14). If $f \in \mathcal{H}_{\alpha}$, then by Result C,

$$
\left|f(x)-\mathcal{B}_{N}^{\chi}(f, x)\right| \leq 2 K \frac{(\Lambda(x))^{\frac{\alpha}{2}}}{N^{\frac{\alpha \beta}{2}}} .
$$

If $f^{\prime} \in \mathcal{H}_{\alpha}$, then by (19) and by property 3 of moduli of smoothness,

$$
\left|f(x)-\mathcal{B}_{N}^{\chi}(f, x)\right| \leq K\left(\frac{\Lambda(x)}{N^{\beta}}\right)^{\frac{1+\alpha}{2}}, \quad x \in[0,1] .
$$

From the mean and the variance of the binomial and hypergeometric distributions, one gets for the classical Bernstein operator $\lambda_{N}(x)=x(1-x) / N$, and for the "hypergeometric" operator

$$
\lambda_{N}(x)=\frac{x(1-x)}{N}\left(1-\frac{N-1}{M-1}\right) \leq \frac{x(1-x)}{N} .
$$

In the next section we apply the results of this section to the approximation of SVFs with convex compact images.

\section{Set-valued functions with convex images - approximations with Minkowski} averages. Let $F:[0,1] \rightarrow \mathcal{C}_{n}$ be a given SVF with convex compact images. For the given data points in $[0,1], 0 \leq t_{0}<t_{1}<\ldots<t_{N} \leq 1$, consider the approximating operator (2) with $C_{i, N}(t) \geq 0$ and with Minkowski sums of sets replacing addition of numbers. Clearly, $\mathcal{A}_{N}$ satisfies

$$
\mathcal{A}_{N}(\lambda F+\mu G, \cdot)=\lambda \mathcal{A}_{N}(F, \cdot)+\mu \mathcal{A}_{N}(G, \cdot), \quad \lambda, \mu \geq 0 .
$$

Moreover, by the positivity of $C_{i, N}(t)$, the images of $\mathcal{A}_{N}(F, \cdot)$ remain in the cone $\mathcal{C}_{n}$. Also, if $\mathcal{A}_{N}$ preserves monotonicity of real-valued functions, then $\mathcal{A}_{N}$ preserves set-inclusion of SVFs.

The approximation property of such operators, as well as their shape-preservation properties, follow from the parametrization of convex compact sets by their support function. The following properties of the support functions $\delta^{*}$ are well known ([15]).

For $A, B \in \mathcal{C}_{n}$,

1. $\delta^{*}(A+B, \cdot)=\delta^{*}(A, \cdot)+\delta^{*}(B, \cdot)$,

2. $\delta^{*}(\lambda A, \cdot)=\lambda \delta^{*}(A, \cdot), \quad \lambda \geq 0$, 
3. $A \subseteq B \Longleftrightarrow \delta^{*}(A, l) \leq \delta^{*}(B, l) \quad$ for each $l \in \mathbb{R}^{n}$,

4. haus $(A, B)=\max _{l \in S_{n-1}}\left|\delta^{*}(A, l)-\delta^{*}(B, l)\right|$.

Thus, for the operator $\mathcal{A}_{N}$ in (2),

$$
\delta^{*}\left(\mathcal{A}_{N}(F, t), l\right)=\mathcal{A}_{N}\left(\delta^{*}(F, l), t\right), \quad l \in \mathbb{R}^{n} .
$$

Also, $F(\cdot) \in \mathcal{H}_{\alpha}$ iff $\delta^{*}(F(\cdot), l) \in \mathcal{H}_{\alpha}$, uniformly in $l \in S_{n-1}$. The above two observations enable to extend approximation results for positive operators from the case of real-valued functions to the case of set-valued functions with compact convex images.

Schoenberg operators. In analogy to (3), we define for a SVF $F(\cdot)$, the set-valued Schoenberg spline approximation of degree $m$ by

$$
S_{m}^{N}(F, t)=\sum_{i=0}^{N} B_{m}(N t-i) F\left(\frac{i}{N}\right), \quad \tilde{S}_{m}^{N}(F, t)=\sum_{i=0}^{N} \tilde{B}_{m}(N t-i) F\left(\frac{i}{N}\right),
$$

where $\Sigma$ stands for a Minkowski sum of sets.

Applying (7),(8),(10) to the support functions $\delta^{*}(F(t), l)$, we conclude from (23) that THEOREM 4.1. (a) If $F \in \mathcal{H}_{\alpha}$, then

$$
\operatorname{haus}\left(F(t), S_{m}^{N}(F, t)\right)=O\left(1 / N^{\alpha}\right) \quad \text { and } \operatorname{haus}\left(F(t), \tilde{S}_{m}^{N}(F, t)\right)=O\left(1 / N^{\alpha}\right) .
$$

(b) If the support function $\delta^{*}(F(t), l)$, as a function of $t$, has first derivative in $\mathcal{H}_{\alpha}$, uniformly in $l \in S_{n-1}$, then

$$
\operatorname{haus}\left(F(t), \tilde{S}_{m}^{N}(F, t)\right)=O\left(\frac{1}{N^{1+\alpha}}\right) .
$$

Spline subdivision schemes. Consider the spline subdivision schemes given by (11) with Minkowski sums of sets replacing addition. Thus for $F:[0,1] \rightarrow \mathcal{C}_{n}$ we take $F_{i}^{0}=$ $F\left(\frac{i}{N}\right), i=0,1, \ldots, N$, and define $F_{i}^{0}=\{0\}$ for $i \in Z \backslash\{0,1, \ldots, N\}$. The subdivision refinement step has the form

$$
F_{i}^{k}=\sum_{j \in Z} a_{i-2 j}^{[m]} F_{j}^{k-1}, i \in Z, \quad k=1,2, \ldots
$$

At the $k$-th refinement level $(k \geq 0)$ we construct as in (12) a piecewise-linear SVF

$$
F^{[k]}(t)=\sum_{i \in Z} F_{i}^{k} \tilde{B}_{1}\left(2^{k} t-i\right), \quad t \in R .
$$

Using (12) for the data generated by the subdivision scheme applied to the samples of $\delta^{*}\left(F^{[0]}(\cdot), l\right)$, the following set-valued analog of (13) is obtained [9]:

THEOREM 4.2. The set-valued functions $\left\{F^{[k]}(\cdot)\right\}_{k=1}^{\infty}$ converge uniformly on any compact subset of $R$ to the $S V F$

$$
F^{\infty}(t)=S_{m}^{N}\left(F, N^{-1} t\right), \quad t \in R .
$$

This implies that in the case of SVFs with convex images, similarly to the case of realvalued functions, the Schoenberg operators are equivalent to the limit of the corresponding spline subdivision schemes performed with Minkowski sums of sets.

Moreover, set inclusion and convexity of the graph are preserved by the spline subdivision schemes $[9]$. 
Bernstein-type approximations. The Bernstein-type approximation $\mathcal{B}_{N}^{\chi}(F, \cdot):[0,1] \rightarrow \mathcal{K}_{n}$ of $F$, defined as in (2) with Minkowski sums of sets, has the form

$$
\mathcal{B}_{N}^{\chi}(F, t)=\sum_{i=0}^{N} P\left(\chi_{t}^{N}=i\right) F\left(\frac{i}{N}\right),
$$

Applying (20), (21) for the support function $\delta^{*}(F(\cdot), l)$, we get

THEOREM 4.3 (cf. [18], [11]). Let $\lambda_{N}(x)$ be defined as in (18) and satisfy $\lambda_{N}(x) \leq$ $\Lambda(x) / N^{\beta}$. If $F \in \mathcal{H}_{\alpha}$, then

$$
\operatorname{haus}\left(F(x), \mathcal{B}_{N}^{\chi}(F, x)\right) \leq 2 K \frac{\Lambda(x)^{\frac{\alpha}{2}}}{N^{\frac{\alpha \beta}{2}}} .
$$

If $\frac{d}{d t} \delta^{*}(F(\cdot), l) \in \mathcal{H}_{\alpha}$, uniformly in $l \in S_{n-1}$, then

$$
\operatorname{haus}\left(F(x), \mathcal{B}_{N}^{\chi}(F, x)\right) \leq K\left(\frac{\Lambda(x)}{N^{\beta}}\right)^{\frac{1+\alpha}{2}}, \quad x \in[0,1] .
$$

Note that the error estimates for the classical Bernstein approximation and the "hypergeometric" approximation of set-valued functions with convex compact images are given by (29) and (30) with $\beta=1, \Lambda(x)=x(1-x)$.

REMARK 4.4. Moduli of smoothness of SVFs with compact convex images are defined in [7] in terms of the corresponding moduli of their support functions. Analogs of results $\mathrm{A}, \mathrm{B}$ and $\mathrm{C}$ for the approximation of set-valued functions with convex compact images can be obtained in terms of these moduli. See also [9] for the use of finite differences of convex compact sets to the study of shape-preserving properties of the spline subdivision schemes.

5. The non-convex case. It was noticed by Vitale [18] that the piecewise-linear approximation constructed with Minkowski sums does not converge to the constant SVF $F(t)=\{0,1\}$ with non-convex images in $R$. Indeed, if $F(t) \equiv A$ with $A \subset R^{n}$ a compact, non-convex set, then, as in (1),

$$
F(t) \subset \tilde{S}_{m}^{N}(F, t)=S_{m}^{N}(F, t), \quad F(t) \neq \tilde{S}_{m}^{N}(F, t) .
$$

For approximation operators of the form (2), as the Bernstein-type operators, where the number of summands grows with $N$, the Shapley-Folkman-Starr Theorem (see Appendix 2 in [17] and Theorem 2 in [5]) yields

$$
\operatorname{haus}\left(\mathcal{A}_{N}(F, t), \operatorname{co}_{N}(F, t)\right) \leq \sqrt{n} \max _{0 \leq i \leq N} C_{i, N}(t) \max _{s \in[0,1]}|F(s)|,
$$

for any SVF with compact images in $R^{n}$. Note that $\operatorname{co} \mathcal{A}_{N}(F, t)=\mathcal{A}_{N}(\operatorname{co} F, t)$ and that by the results of the previous section $\lim _{N \rightarrow \infty} \mathcal{A}_{N}(\operatorname{co} F, t)=\operatorname{co} F(t)$. If $\lim _{N \rightarrow \infty} \max \left\{C_{i, N}(t)\right.$ : $0 \leq i \leq N\}=0$, then $\lim _{N \rightarrow \infty} \mathcal{A}_{N}(F, t)=\operatorname{co} F(t)$. As a consequence of this observation, Bernstein-type operators generated by discrete random variables satisfying $\lim _{N \rightarrow \infty} \max \left\{P\left(\chi_{t}^{N}=i\right): 0 \leq i \leq N\right\}=0$, when applied to a SVF, $F(t)$, with compact images in $R^{n}$, converge to the convex hull of $F(t)$. In particular, this convexification phenomenon is observed for the Bernstein operators and the "hypergeometric" operators [18], [11]. 
The convexification of the images of the sets obtained by spline subdivision schemes was proved in [11]. In this case the Shapley-Folkman-Starr Theorem is not applicable since subdivision schemes are recursive averaging procedures with a fixed finite number of summands and fixed weights. For this case an inequality, involving a measure of nonconvexity of sets, proved in [5], is invoked to prove that spline subdivision schemes with Minkowski sums applied to arbitrary initial compact sets in $R^{n}$ converge to a SVF with convex images.

Note that the direct calculation of the set-valued spline functions (24) is not necessarily convexifying, since only a fixed number of Minkowski averages are calculated. This differs from the case of subdivision schemes, which are limiting procedures involving an unbounded number of Minkowski averages.

5.1. Approximation with metric averages. The lack of approximation by the operators of Section 4 in case of SVFs with general images is due to the fact that the Minkowski averages of non-convex sets are too big. To obtain approximating operators in the nonconvex case we apply an operation of averaging between two sets which yields a subset of the Minkowski average.

This operation between two compact sets was introduced in [1] for the construction of piecewise-linear approximation of SVFs with compact (not necessarily convex) images. The same operation was used in [10] for the construction of set-valued subdivision schemes which are also approximating in the non-convex case. Although this operation, called in [10] "metric average", does not possess some essential properties of the Minkowski sum, it has several properties which make it appropriate for our purposes.

Definition 5.1. Let $A, B \in \mathcal{K}_{n}$ and $0 \leq t \leq 1$. The $t$-weighted metric average of $A$ and $B$ is

$$
A \oplus_{t} B=\left\{t\{a\}+(1-t) \Pi_{B}(a): a \in A\right\} \cup\left\{t \Pi_{A}(b)+(1-t)\{b\}: b \in B\right\},
$$

where the linear combinations above are in the Minkowski sense.

The following properties of the metric average are easy to observe [10].

Let $A, B, C \in \mathcal{K}_{n}$ and $0 \leq t \leq 1,0 \leq s \leq 1$. Then

1. $A \oplus_{0} B=B, \quad A \oplus_{1} B=A, \quad A \oplus_{t} B=B \oplus_{1-t} A$.

2. $A \oplus_{t} A=A$.

3. $A \cap B \subseteq A \oplus_{t} B \subseteq t A+(1-t) B$.

The metric property of this average [1] is essential for our applications :

4. $\operatorname{haus}\left(A \oplus_{t} B, A \oplus_{s} B\right)=|t-s| \operatorname{haus}(A, B)$.

The main disadvantage of the metric average, as an operation on sets, is the lack of associativity, which is the reason why it is hard to extend this binary operation to an average of three or more sets. Thus we need to represent the positive operators in terms of repeated binary averages. This is not hard for the Schoenberg spline approximation, for the spline subdivision schemes and for the Bernstein approximation. Let us note that the representation of these operators in terms of repeated binary averages is non-unique [19]. The representations chosen here are successful computationally for real-valued functions $[4,14]$ and proved to be adequate theoretically and experimentally for SVFs $[10,13]$. 
5.2. Metric Schoenberg spline approximation. For the Schoenberg operators we use a representation in terms of repeated binary averages based on the recurrence formula for B-splines ([4], chapter 10),

$$
B_{m+1}(t)=\frac{t}{m+1} B_{m}(t)+\frac{m+1-t}{m+1} B_{m}(t-1),
$$

where $B_{m}(\cdot)$ is the B-spline of degree $m$ defined in Section 3.1. This yields the following algorithm for the evaluation of $S_{m}(t)=\sum_{i} a_{i} B_{m}(t-i)$ in terms of repeated binary averages: For $t \in[j, j+1)$,

$$
S_{m}(t)=\sum_{i=j-m+k}^{j} a_{i}^{k}(t) B_{m-k}(t-i), \quad k=0,1, \ldots, m,
$$

with

$$
\begin{gathered}
a_{i}^{0}(t)=a_{i}, \quad i=j-m, \ldots, j, \\
a_{i}^{k}(t)=\frac{i+m+1-k-t}{m+1-k} a_{i-1}^{k-1}(t)+\frac{t-i}{m+1-k} a_{i}^{k-1}(t), \quad i=j-m+k, \ldots, j .
\end{gathered}
$$

In particular, $S_{m}(t)=a_{j}^{m}(t)$.

We apply the above recurrence formulae to define $S_{m}^{N}(F, t)$ for $t \in[j, j+1)$ procedurally by

$$
\begin{aligned}
F_{i}^{0} & =F\left(\frac{i}{N}\right), & i & =j-m, \ldots, j, \\
F_{i}^{k} & =F_{i-1}^{k-1} \oplus_{\lambda_{i}^{k}} F_{i}^{k-1}, & i & =j-m+k, \ldots, j, \quad k=1, \ldots, m,
\end{aligned}
$$

with

$$
\lambda_{i}^{k}=\frac{i+m+1-k-N t}{m+1-k}, \quad i=j-m+k, \ldots, j, \quad k=1, \ldots, m,
$$

and, as above, determine

$$
S_{m}^{N}(F, t)=F_{j}^{m} .
$$

TheOREm 5.2 ([13]). Let the set-valued function $F:[a, b] \rightarrow \mathcal{K}_{n}$ be Hölder continuous with exponent $\alpha, \alpha \in(0,1]$. Then $S_{m}^{N}(F, t)$ defined by (35)-(38) satisfies

$$
\operatorname{haus}\left(S_{m}^{N}(F, t), F(t)\right) \leq \frac{C}{N^{\alpha}} .
$$

5.3. Metric spline subdivision schemes. To compute the $m$-th degree spline subdivision scheme in terms of metric averages, we represent it by a sequence of repeated binary averages.

The refinement step (11) can be obtained by one step of refinement of the linear spline subdivision, followed by $m-1$ binary averages. Adapting this representation to sets, the sequence of steps which replaces (25) consists of first defining

$$
F_{2 i+1}^{k+1,0}=F_{i}^{k}, \quad F_{2 i}^{k+1,0}=F_{i-1}^{k} \oplus_{\frac{1}{2}} F_{i}^{k}, \quad i \in Z,
$$

and then calculating recursively for $1 \leq j \leq m$ the intermediate averages

$$
F_{i}^{k+1, j}=F_{i}^{k+1, j-1} \oplus_{\frac{1}{2}} F_{i-1}^{k+1, j-1} .
$$

The values at level $k+1$ are

$$
F_{i}^{k+1}=F_{i}^{k+1, m-1} \quad, i \in Z .
$$


At the $k+1$-the refinement level, a metric piecewise-linear SVF, $F^{[k+1]}(t)$, is constructed in analogy to (26),

$$
F^{[k+1]}(t)=F_{i}^{k+1} \oplus_{\lambda(t)} F_{i+1}^{k+1}, \quad i 2^{-(k+1)} \leq t \leq(i+1) 2^{-(k+1)}, \quad i \in Z
$$

where $\lambda(t)=(i+1)-t 2^{k+1}$.

The following claims are proved in [10].

THEOREM 5.3. The sequence $\left\{F^{[k]}(\cdot)\right\}_{k \in Z_{+}}$converges uniformly to a set-valued function $F^{\infty}(\cdot)$. If $L=\sup \left\{\operatorname{haus}\left(F_{\alpha}^{0}, F_{\alpha+1}^{0}\right): \alpha \in Z\right\}$, then $F^{\infty}$ is Lipschitz continuous with Lipschitz constant $L$.

THEOREM 5.4. Let the set-valued function $F(\cdot): R \rightarrow \mathcal{K}_{n}$ be Lipschitz continuous with a Lipschitz constant $L$, let the initial sets be given by $F_{i}^{0}=F\left(\frac{i}{N}\right), i \in Z$, and let $F^{\infty}(\cdot)$ be as in Theorem 5.3. Then

$$
\max _{t} \operatorname{haus}\left(F^{\infty}(N t), F(t)\right) \leq \frac{(7+m) L}{2 N}
$$

REMARK 5.5. The estimate in (43) can be modified to $O\left(N^{-\alpha}\right)$ for $F \in \mathcal{H}_{\alpha}$.

Theorem 5.4 guarantees that $F^{\infty}(N \cdot)$ approximates $F(\cdot)$, also if $F$ has non-convex images. Thus the subdivision defined by (40)-(42) is not convexifying, in contrast to the subdivision based on Minkowski averages.

REMARK 5.6. Theorem 4.4 and Corollary 4.5 in [10] should be corrected, replacing $F^{k}(t)$ and $F^{\infty}(t)$ there by $F^{k}(t / h)$ and $F^{\infty}(t / h)$ respectively.

5.4. Metric Bernstein approximation. For $f$ a real-valued function, the value $B_{N}(f, t)$ can be calculated recursively by using the de Casteljau algorithm, in terms of repeated binary averages [14]. The algorithm is based on the recurrence relation

$$
C_{i, N}(t)=(1-t) C_{i, N-1}(t)+t C_{i-1, N-1}(t),
$$

where $C_{i, N}(t)=\left(\begin{array}{c}N \\ i\end{array}\right) t^{i}(1-t)^{N-i}$ are the basic Bernstein polynomials.

For $t \in[0,1], B_{N}(f, t)$ in (16) can be presented by a repeated application of (44) as

$$
B_{N}(f, t)=\sum_{i=0}^{N-k}\left(\begin{array}{c}
N-k \\
i
\end{array}\right) t^{i}(1-t)^{N-k-i} f_{i}^{k}, \quad k=0,1, \ldots, N,
$$

with the values $f_{i}^{k}$ given recursively by

$$
f_{i}^{k}=(1-t) f_{i}^{k-1}+t f_{i+1}^{k-1}, \quad i=0,1, \ldots, N-k, k=1, \ldots, N,
$$

and with $f_{i}^{0}=f(i / N), i=0,1, \ldots, N$. Obviously $B_{N}(f, t)=f_{0}^{N}$.

For a SVF $F(\cdot)$ this algorithm in terms of repeated metric averages of compact sets is: Starting with $F_{i}^{0}=F\left(\frac{i}{N}\right), i=0,1, \ldots, N$, and a fixed $t \in[0,1]$, calculate

$$
F_{i}^{k}=F_{i}^{k-1} \oplus_{1-t} F_{i+1}^{k-1}, \quad i=0,1, \ldots, N-k, k=1, \ldots, N,
$$

and define

$$
B_{N}(F, t)=F_{0}^{N} .
$$

This is a procedural definition of $B_{N}(F, t)$. 
It is not known whether $B_{N}(F, \cdot)$ converges to $F(\cdot)$ for $F$ with general compact images in $R^{n}$, except for the case of set-valued functions with images in $R$ all of the same topology [13]. The proof of the convergence relies on a measure of minimal separation between the components of a compact set $A \subset R$. If $A$ consists of a number of disjoint ordered intervals (some possibly with an empty interior): $A=\bigcup_{j=1}^{J} A_{j}$ with $a_{j}<a_{j+1}$ for any $a_{j} \in A_{j}$ and $a_{j+1} \in A_{j+1}, j=1, \ldots, J-1$., the measure of minimal separation between the components of $A$ is defined by

$$
s(A)=\inf _{l, j \in\{1, \ldots, J\}, l \neq j}\left\{\operatorname{dist}\left(a, A_{j}\right): a \in A_{l}\right\}
$$

THEOREM 5.7. Let the set-valued function $F:[0,1] \rightarrow \mathcal{K}_{1}$ be Lipschitz continuous, such that for each $t, F(t)=\bigcup_{j=1}^{J} F_{j}(t)$, where $\left\{F_{j}(t)\right\}$ are disjoint ordered intervals such that $s^{*}=\inf _{0 \leq t \leq 1} s(F(t))>0$. Then for $N$ large enough

$$
\operatorname{haus}\left(B_{N}(F, t), F(t)\right) \leq \widetilde{C} / \sqrt{N}, \quad t \in[0,1] .
$$

In conclusion, the Schoenberg metric operators defined in section 5 in two ways are approximating operators for SVFs with general compact images, while the Schoenberg operators defined in terms of Minkowski averages are approximating only when applied to SVFs with convex images. Yet, the question whether the metric Bernstein operators defined in terms of the de Casteljau algorithm are approximating, is open.

In our future work we intend to adapt approximating operators to SVFs extending the metric average to more than two sets. With such an operation on sets, the representation of the operators in terms of repeated binary averages is not needed anymore. It seems that in this new adaptation many approximating operators for real-valued functions, including the Bernstein operators, become approximating operators for SVFs, yet their evaluation may be much more complicated [12].

Acknowledgements. The authors acknowledge the support of the Israel Science Foundation - Center of Excellence Program, the Hermann Minkowski Center for Geometry, and the Internal Research Foundation at Tel-Aviv University.

\section{References}

[1] Z. Artstein, Piecewise linear approximations of set-valued maps, Journal of Approx. Theory 56 (1989), 41-47.

[2] R. Baier, Mengenwertige Integration und die diskrete Approximation erreichbarer Mengen, Bayreuth. Math. Schr. 50, 1995.

[3] R. Baier, N. Dyn and E. Farkhi, Metric averages of one dimensional compact sets, in: Approximation Theory X, C. Chui, L. L. Schumaker and J. Stoeckler (eds.), Vanderbilt Univ. Press, Nashville, TN, 2002, 9-22.

[4] C. de Boor, A Practical Guide to Splines, Springer-Verlag, New York, 1978.

[5] J. W. S. Cassels, Measures of the non-convexity of sets and the Shapley-Folkman-Starr theorem, Math. Proc. Camb. Phil. Soc. (1975), 78, 433-436.

[6] R. DeVore and G. Lorentz, Constructive Approximation, Springer-Verlag, Berlin, 1993.

[7] T. Donchev and E. Farkhi, Moduli of smoothness of vector-valued functions of a real variable and applications, Numer. Funct. Anal. Optimiz. 11 (1990), 497-509. 
[8] N. Dyn, Subdivision schemes in computer-aided geometric design, in: Advances in Numerical Analysis, Vol. II, Wavelets, Subdivision Algorithms and Radial Basis Functions, W. Light (ed.), Clarendon Press, Oxford, 1992, 36-104.

[9] N. Dyn and E. Farkhi, Spline subdivision schemes for convex compact sets, Journal of Comput. Appl. Mathematics 119 (2000), 133-144.

[10] N. Dyn and E. Farkhi, Spline subdivision schemes for compact sets with metric averages, in: Trends in Approximation Theory, K. Kopotun, T. Lyche and M. Neamtu (eds.), Vanderbilt Univ. Press, Nashville, TN, 2001, 95-104.

[11] N. Dyn and E. Farkhi, Set-valued approximations with Minkowski averages - convergence and convexification rates, Numer. Funct. Anal. Appl. 25 (2004), 363-377.

[12] N. Dyn, E. Farkhi and A. Mokhov, Approximations of set-valued functions by metric linear operators, Constr. Approx., to appear.

[13] N. Dyn and A. Mokhov, Approximations of set-valued functions based on the metric average, Rend. Mat. Appl., to appear.

[14] H. Prautzsch, W. Boehm and M. Paluszny, Bezier and B-Spline Techniques, Springer, 2002.

[15] R. T. Rockafellar, Convex Analysis, Princeton University Press, Princeton, 1970.

[16] R. Schneider, Convex Bodies: The Brunn-Minkowski Theory, Cambridge University Press, Cambridge, 1993.

[17] R. Starr, Quasi-equilibria in markets with non-convex preferences, Econometrica 37 (1969), $25-38$.

[18] R. Vitale, Approximation of convex set-valued functions, J. Approximation Theory 26 (1979), 301-316.

[19] J. Wallner and N. Dyn, Convergence and $C^{1}$ analysis of subdividision schemes on manifolds by proximity, CAGD 22 (2005), 593-622. 